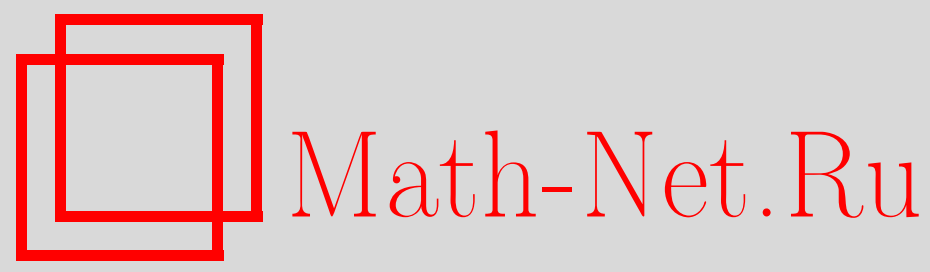

А. Г. Шухов, О зависимости показателя роста от длины определяющего соотношения, Матем. заметки, 1999, том 65, выпуск 4, 612-618

DOI: https://doi.org/10.4213/mzm1088

Использование Общероссийского математического портала Math-Net.Ru подразумевает, что вы прочитали и согласны с пользовательским соглашением http://www . mathnet.ru/rus/agreement

Параметры загрузки:

IP : 52.205 .19 .152

26 апреля 2023 г., 18:05:00

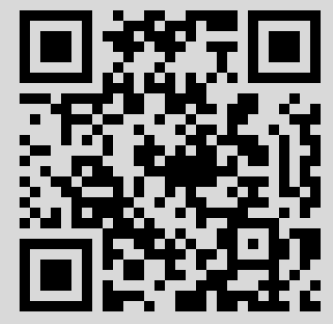




\section{О ЗАВИСИМОСТИ ПОКАЗАТЕЛЯ РОСТА ОТ ДЛИНЫ ОПРЕДЕЛЯЮШЕГО СООТНОШЕНИЯ}

\section{А. Г. Шухов}

Пусть $G_{k}=\left\langle a_{1}, \ldots, a_{m} \mid r_{1}^{(k)}=\cdots=r_{j_{k}}^{(k)}=1\right\rangle=\left\langle A \mid R_{k}\right\rangle-$ последовательность конечно определенных групп с порождающим множеством $A=\left\{a_{1}, \ldots, a_{m}\right\}$, $R_{k}$ - симметризованное множество слов над алфавитом $A \cup A^{-1}$, полученное из определяющих слов и их обратных всеми циклическими сдвигами. Будем предполагать, что слова из $R_{k}$ являются циклически неприводимьпи, а их длина стремится к $\infty$ с ростом $k$. В работе доказано, что если $R_{k}$ удовлетворяют условию малого сокращения $C^{\prime}(1 / 6)$ и число соотношений не очень быстро растет с ростом $k$, то показатель роста $\lambda\left(G_{k}\right) \rightarrow 2 m-1$ при $k \rightarrow \infty$.

Библиографофия: 5 названий.

1. Формулировка результата. Пусть $G$-группа с конечньм множеством $A=$ $\left\{a_{1}, \ldots, a_{m}\right\}$ ее порождаюших. Для элемента $g \in G$ длиной $|g|$ будем назьвать минимальное число элементов из $A \cup A^{-1}$, необходимых для записи $g$. Обозначим через $\lambda=\lambda(G)$ показатель роста:

$$
\lambda(G)=\lim _{n \rightarrow \infty} \sqrt[n]{\gamma(n)}
$$

где $\gamma(n)=\#\{g \in G:|g| \leqslant n\}-$ число элементов длины, не превосходящей $n$. Функция $\gamma(n)$ назьвается функиией роста әруппь $G$.

В работе [1] поставлена следующая задача: при каких условиях

$$
\lim _{k \rightarrow \infty} \lambda\left(G_{k}\right)=2 m-1
$$

где $G_{k}=\left\langle a_{1}, \ldots, a_{m} \mid r^{(k)}=1\right\rangle$ - последовательность групш с $m$ образуюшими и одним определяюшим соотношением, длина которого с ростом $k$ стремится к $\infty$. Напомним, что $2 m-1$ - показатель роста свободной групшы с $m$ образующими. В [1] отмечено, что в общем случае равенство (1) не имеет места (см. пример ниже).

Цель настоящей заметки - сформулировать некоторые достаточные условия, при которых равенство (1) справедливо.

Пусть $G=\left\langle a_{1}, \ldots, a_{m} \mid r_{1}=\cdots=r_{j}=1\right\rangle=\langle A \mid R\rangle$ - конечно определенная группа, $R$ - симметризованное множество слов над алфавитом $A \cup A^{-1}$, полученное из определяющих слов $r_{i}$ и их обратных $r_{i}^{-1}$ всеми циклическими сдвигами. Будем предполагать, что слова $r \in R$ являются циклически неприводимыми.

Работа выполнена при финансовой поддержке Российского фонда фундаментальых исследований, грант № 96-01-00974, и фонда INTAS, грант № 94-320. 
Слово $w$ называется куском относительно $R$, если существуют два различных элемента из $R$ вида $w v$ и $w v^{\prime}$, т.е. кусок - это слово, которое сокращается в произведении двух различных элементов из $R$.

Пусть $\lambda>0$. Множество $R$ удовлетворяет условию $C^{\prime}(\lambda)$, если $|w|<\lambda|r|$ для любого $r \in R$ и каждого его подслова $w$, которое является куском относительно $R$ (см. [2], [3]).

Пусть $G_{k}=\left\langle a_{1}, \ldots, a_{m} \mid r_{1}^{(k)}=\cdots=r_{j_{k}}^{(k)}=1\right\rangle=\left\langle A \mid R_{k}\right\rangle$ - последовательность $m$-порожденных групп с циклически неприводимыми определяющими соотношениями $r_{j}^{(k)}, j=1, \ldots, j_{k}$. Обозначим через $l_{k}$ и $L_{k}$ длины самого короткого и самого длинного слова в $R_{k}$, и пусть $t_{k}=\left[l_{k} / 6\right]$.

Теорема. Пусть $G_{k}=\left\langle A \mid R_{k}\right\rangle$ и симметризованные мнохсества $R_{k}$ удовлетворяют условию $C^{\prime}(1 / 6)$. Предположим, что с ростом $k$ длина самого короткого определяюшего соотношения $l_{k}$ стремится $\kappa \infty u$

$$
\lim _{k \rightarrow \infty} j_{k} L_{k}\left(\frac{2}{2 m-1}\right)^{t(k)}=0
$$

Тогда имеет место равенство (1).

СледствИЕ. Если $G_{k}$ - последовательность -порожденных групп $c$ одним определяющим соотношением, удовлетворяющих условию $C^{\prime}(1 / 6)$, то справедливо равенство (1).

Доказательство теоремы разбивается на два этапа: первьй связан с использованием леммы Гриндлингера для $C^{\prime}(1 / 6)$-групп [3], второй основан на технике работы [4].

Нам потребуются два определения. Пусть $G=\langle A \mid R\rangle$. Слово $X$ (над алфавитом $\left.A \cup A^{-1}\right)$ называется $\alpha$-приведенным относительно $R, \alpha \in(0,1)$, если $X$ свободно несократимо и не содержит $\alpha$-части определяющего слова, т.е. $X$ нельзя представить в виде $u_{1} w u_{2}$, где $w$ - подслово некоторого $r \in R,|w| \geqslant[\alpha|r|]$.

В дальнейшем будем предполагать, что $\alpha|r| \geqslant 2$ для любого $r \in R$. Множество $\alpha$-приведенных слов будем обозначать через $\mathscr{N}(\alpha)$.

Слово $Y$ называется геодезическим, если соответствующий этому слову путь (с началом в единичном элементе) в графе Кэли группы $G$ (см., например, [2]) является геодезическим.

\section{2. Доказательство теоремы.}

Лемма 1. Пусть $G=\langle A \mid R\rangle$ и $R$ удовлетворяет условию $C^{\prime}(1 / 6), \mathcal{N}=\mathcal{N}(1 / 6)$ есть мнохество 1/6-приведенных слов относительно $R$. Тогда

1) различные слова из $\mathscr{N}$ представляют различные әлементы из $G$, т.е. если $X, Y$ - разные слова в $\mathscr{N}$, то $X \neq Y$ в группе $G$

2) слова из $\mathscr{N}$ являются геодезическими; более того, если $X \in \mathcal{N}$, а $Y$ - геодезическое слово $(Y$, вообще говоря, не принадлежит $\mathcal{N})$, то из равенства $X=Y$ в $G$ вытекает их графическое равенство.

Доказательство леммы 1 основано на применении следующего утверждения. Для $q>$ 0 и слова $s$ над алфавитом $A \cup A^{-1}$ будем писать $s>q R$, если существует $r \in R$, приведенная форма которого имеет вид $r=s t$ и $|s|>q|r|$. 
ЛЕММА ГРИНДЛИНГЕРА ДЛЯ 1/6-ГРУПП [3]. Пусть $R$ удовлетворяет условию $C^{\prime}(1 / 6)$. Предположим, что $w$ - нетривиальное ииклически приведенное слово такое, что $w \in N(R)$, где $N(R)$ - нормальное замыкание $R$ в свободной группе $F_{m}$ с образуюшими из $A$. Тогда либо

1) $w \in R$,

либо некоторая ииклически приведенная перестановка элемента $w$ содержит одно из следующих:

2) два непересекающихся подслова, каждое из которых > $(5 / 6) R$;

3) три непересекаюшихся подслова, каждое из которых > $(4 / 6) R$;

4) четыре непересекающихся подслова, два из которых > (4/6) $R$ и два $>(3 / 6) R$

5) пять непересекающихся подслов, четыре из которых $>(3 / 6) R$ и одно $>(4 / 6) R$

6) шесть непересекающихся подслов, каждое из которых > (3/6)R.

ДокАЗАТЕЛЬСТво ЛЕммы 1 . Пусть $X, Y$-разные слова в $\mathscr{N}$, но $X=Y$ в $G$. Начала и окончания слов $X$ и $Y$ могут совпадать, т.е. $X=X_{0} X_{1} X_{2}, Y=X_{0} Y_{1} X_{2}$, при этом $X_{1}=Y_{1}$ в $G, X_{1}, Y_{1} \in \mathcal{N}$, и слово $w=X_{1} Y_{1}^{-1}$ циклически приведено. Поскольку $X_{1}$ и $Y_{1}$ принадлежат $\mathscr{N}$, слово $w=X_{1} Y_{1}^{-1}$ (либо некоторая циклическая перестановка $w^{*}$ слова $w)$ может содержать максимум либо два подслова, каждое из которых принадлежит $\mathcal{N}(2 / 6)$, либо одно подслово из $\mathcal{N}(3 / 6)$, что противоречит лемме Гриндлингера. Утверждение 1) леммы 1 доказано.

Пусть теперь $X \in \mathcal{N}, Y$ - геодезическое слово и $X=Y$ в $G$. Как и выше, запишем $X=X_{0} X_{1} X_{2}, Y=X_{0} Y_{1} X_{2}$, где $X_{1} \in \mathscr{N}, Y_{1}$ - геодезическое слово, $w=X_{1} Y_{1}^{-1}$ циклически приведено.

1) Пусть $w \in R$. Поскольку $X_{1} \in \mathcal{N}(1 / 6)$, то $\left|X_{1}\right|<(1 / 6)|w|$ и, следовательно, $\left|Y_{1}\right| \geqslant(5 / 6)|w|$, что противоречит геодезичности $Y_{1}$.

2) Пусть $w$ (либо некоторая циклическая перестановка $w^{*}$ слова $w$ ) содержит два непересекаюшихся подслова, каждое из которых $>(5 / 6) R$. Тогда $Y_{1}$ либо включает два подслова, каждое из которых $>(4 / 6) R$, либо одно подслово $>(5 / 6) R$. И в том, и в другом случае получаем противоречие с геодезичностью $Y_{1}$.

Случаи 3)-6) леммы Гриндлингера рассматриваются аналогично, и лемма 1 доказана.

Лемма 1 позволяет не различать слова из $\mathscr{N}$ над алфавитом $A \cup A^{-1}$ и соответствующие элементы группы $G$, чем мы и будем пользоваться в дальнейшем.

Перейдем ко второму этапу доказательства теоремы. Пусть $\delta(n)=\#\{g \in G$ : $|g|=n\}$ - число элементов группы $G$ длины $n$. В частности, для свободной групшы $F_{m}$ с $m$ образующими $\delta(n)=2 m(2 m-1)^{n-1}(\delta(n)$ назьвается сферической функиией роста). Соответствующая производящая функиия роста

$$
H^{(m)}(z)=\sum_{n=0}^{\infty} \delta(n) z^{n}=1+2 m z \sum_{n=1}^{\infty}(2 m-1)^{n-1} z^{n-1}=1+\frac{2 m z}{1-(2 m-1) z}
$$

Нам потребуется неравенство, аналогичное неравенству Голода-Шафаревича [4]. 
Пусть $U$-некоторое непустое множество свободно несократимых слов над алфавитом $A \cup A^{-1}$, элементы которого будем назьвать запрещенными словами. Слово назьвается допустимым, если оно не содержит вхождений запрещенных слов. Как и в [4], будем считать, что $U$ не содержит слов длины 1 и никакое слово из $U$ не является подсловом другого слова из $U$.

Обозначим через $h(n)$ число допустимых несократимых слов длины $n$, а через

$$
H(z)=\sum_{n=0}^{\infty} h(n) z^{n}
$$

соответствующую производящую функцию. Будем говорить, что

$$
\sum_{n=0}^{\infty} a_{n} z^{n} \geqslant \sum_{n=0}^{\infty} b_{n} z^{n}, \quad \text { если } a_{n} \geqslant b_{n}, \quad n \geqslant 0 .
$$

С учетом этого соглашения имеет место следующее утверждение (cp. с [4, неравенство (11)]).

ЛЕмма 2. Пусть $U$ - множество запрещенных слов над алфавитом $A \cup A^{-1}$, $|A|=m, u H(z)$ - производящая функиия числа допустимых слов. Тогда

а) выполнено

$$
H(z)\left(1-(2 m-1) z+\sum_{u \in U} z^{|u|}\right) \geqslant 1
$$

б) если ряд

$$
\left(1-(2 m-1) z+\sum_{u \in U} z^{|u|}\right)^{-1}
$$

имеет неотрицательные коэффициенты, то

$$
H(z) \geqslant\left(1-(2 m-1) z+\sum_{u \in U} z^{|u|}\right)^{-1} .
$$

ДоКАЗАТЕЛЬСТво. Допустимое слово длины $n+1$ получается из допустимого слова длины $n$ умножением на букву из $A \cup A^{-1}$, не совпадающую с обратным элементом к последней букве данного слова. Число построенных таким способом слов равно $h(n) \times$ $(2 m-1)$. Некоторые из этих слов в конще могут содержать запрещенное слово $u \in U$. Следовательно,

$$
h(n+1) \geqslant h(n) \cdot(2 m-1)-\sum_{u \in U} h(n+1-|u|) .
$$

Умножим это неравенство на $z^{n+1}$ и просуммируем по $n$ :

$$
\begin{aligned}
H(z) & =1+\sum_{n=0}^{\infty} h(n+1) z^{n+1} \\
& \geqslant 1+(2 m-1) z \sum_{n=0}^{\infty} h(n) z^{n}-\sum_{u \in U} \sum_{n=0}^{\infty} z^{|u|} h(n+1-|u|) z^{n+1-|u|} .
\end{aligned}
$$


Следовательно,

$$
H(z) \geqslant 1+(2 m-1) z H(z)-H(z) \sum_{u \in U} z^{|u|}
$$

Отсюда имеем

$$
H(z)\left(1-(2 m-1) z+\sum_{u \in U} z^{|u|}\right) \geqslant 1
$$

и лемма 2 доказана.

Применим лемму 2 в специальном случае. Пусть $\alpha \in(0,1)$ и $G=\langle A \mid R\rangle=$ $\left\langle A \mid r_{1}=\cdots=r_{j}=1\right\rangle$. Симметризованное множество $R$, состоящее из слов, являющихся циклическими сдвигами $r_{i}$ и $r_{i}^{-1}, i=1, \ldots, j$, содержит не более $2\left(\left|r_{1}\right|+\cdots+\left|r_{j}\right|\right)$ элементов. В словах $r_{i}$ и $r_{i}^{-1}, i=1, \ldots, j$, существует $\leqslant 2\left|r_{i}\right|$ подслов длины $\left[\alpha\left|r_{i}\right|\right]$; обозначим их $u_{1}^{i}, \ldots, u_{m_{i}}^{i}$, где $m_{i} \leqslant 2\left|r_{i}\right|$. Назовем $U=\left\{u_{1}^{1}, \ldots, u_{m_{1}}^{1}, \ldots, u_{1}^{j}, \ldots, u_{m_{j}}^{j}\right\}$ множеством запрещенных слов.

Множество допустимых слов в этом случае совпадает с множеством $\mathscr{N}(\alpha) \alpha$-приведенных слов. Обозначим через $h_{\alpha}(n)$ число несократимых слов из $\mathcal{N}(\alpha)$ длины $n$. Пусть

$$
H_{\alpha}(z)=\sum_{n=0}^{\infty} h_{\alpha}(n) z^{n}
$$

- соответствующая производящая функция.

Лемма 3. Пусть $G=\left\langle A \mid r_{1}=\cdots=r_{j}=1\right\rangle$. Тогда

а) выполнено

$$
H_{\alpha}(z)\left(1-(2 m-1) z+\sum_{i=1}^{j}\left|r_{i}\right| z^{\left[\alpha\left|r_{i}\right|\right]}\right) \geqslant 1
$$

б) при $\alpha=1 / 6$ для показателя роста $\lambda(G)$ группы $G$, удовлетворяюшей условию $C^{\prime}(1 / 6)$, имеет место оценка снизу

$$
\lambda(G) \geqslant \xi^{-1}
$$

əде $\xi-$ минимальный положсительный корень многочлена

$$
\varphi(z)=1-(2 m-1) z+2 \sum_{i=1}^{j}\left|r_{i}\right| z^{\left[\left|r_{i}\right| / 6\right]} .
$$

(Напомним, что неравенство (4) необходимо понимать в смысле (3).) 
ДокАЗАТЕльство. Неравенство (4) непосредственно вытекает из леммы 2. Обозначим через $\beta$ радиус сходимости ряда Маклорена функции $H_{1 / 6}(z)$. Заметим, что $\beta \leqslant \xi$; в противном случае неравенство (4) невыполняется в некотором интервале $(\xi, \xi+\delta)$, где $\delta>0$. Для любого натурального $n$ в силу леммы 1 имеем неравенство $\delta(n) \geqslant h_{1 / 6}(n)$, где $\delta(n)$ - число элементов групшы $G$ длины $n$. Отсюда для показателя роста $\lambda(G)$ группы $G$ вытекает оценка

$$
\lambda(G)=\lim _{n \rightarrow \infty} \sqrt[n]{\gamma(n)}=\lim _{n \rightarrow \infty} \sqrt[n]{\delta(n)} \geqslant \lim _{n \rightarrow \infty} \sqrt[n]{h_{1 / 6}(n)}=\frac{1}{\beta} \geqslant \frac{1}{\xi}
$$

и лемма 3 доказана.

Применим лемму 3 к последовательности $G_{k}=\left\langle A \mid r_{1}^{(k)}=\cdots=r_{j_{k}}^{(k)}=1\right\rangle$. Пусть $\xi_{k}$ - минимальный положительньй корень многочлена

$$
\varphi_{k}(z)=1-(2 m-1) z+2 \sum_{i=1}^{j_{k}}\left|r_{i}^{(k)}\right| z^{\left[\left|r_{i}^{(k)}\right| / 6\right]}
$$

В силу леммы $3 \lambda\left(G_{k}\right) \geqslant \xi_{k}^{-1}$. Покажем, что $\xi_{k} \rightarrow(2 m-1)^{-1}$ при $k \rightarrow \infty$. В самом деле, для любого $\varepsilon \in(0,1)$ имеем

$$
\varphi_{k}\left(\frac{1+\varepsilon}{2 m-1}\right)=-\varepsilon+2 \sum_{i=1}^{j_{k}}\left|r_{i}^{(k)}\right|\left(\frac{1+\varepsilon}{2 m-1}\right)^{\left[\left|r_{i}^{(k)}\right| / 6\right]}
$$

Из условия (2) следует, что

$$
\varphi_{k}\left(\frac{1+\varepsilon}{2 m-1}\right)<0
$$

для достаточно большого $k$. Поскольку $\varphi_{k}(z)>0$ при $0 \leqslant z \leqslant(2 m-1)^{-1}$, минимальный положительньй корень $\xi_{k}$ многочлена $\varphi_{k}(z)$ (для достаточно большого $k$ ) принадлежит интервалу

$$
\left(\frac{1}{2 m-1}, \frac{1+\varepsilon}{2 m-1}\right) \text {. }
$$

Поскольку $\lambda\left(G_{k}\right)$ не превосходит показателя свободной группы, т.е. $\lambda\left(G_{k}\right) \leqslant 2 m-1$, то из $(5)$ вытекает, что $\lambda\left(G_{k}\right) \rightarrow 2 m-1$ при $k \rightarrow \infty$, и теорема доказана.

ЗАмЕчАнИЕ. Утверждение теоремы остается справедливым, если условие $C^{\prime}(1 / 6)$ заменить на условие $C^{\prime}(1 / 4)$ и $T(4)$, а константу $\alpha=1 / 6$ заменить на $\alpha=1 / 4$.

В этом случае вместо леммы Гриндлингера используется

Теорема [3, теорема 4.6]. Если $R$ удовлетворяет условию $C^{\prime}(1 / 4)$ u $T(4), w-$ нетривиальное ииклически приведенное слово из $N(R)$, то либо $w \in R$, либо ииклическая перестановка $w^{*}$ слова $w$ содерэит два непересекающихся подслова, каждое из которых > (3/4)R, или четыре непересекающихся подслова, каждое из которых $>(1 / 2) R$.

Напомним, что $R$ удовлетворяет условию $T(q)$ (см. [3]) для натурального q, если для любого $h, 3 \leqslant h \leqslant q$, и элементов $r_{1}, \ldots, r_{h}$ из $R$ таких, что последовательные элементы $r_{i}, r_{i+1}$ не являются взаимно обратными, по крайней мере одно из произведений $r_{1} r_{2}, \ldots, r_{h-1} r_{h}, r_{h} r_{1}$ приведено. 
В заключение приведем пример, показьвающий, что в общем случае равенство (1) не имеет места.

Пусть $G_{k}=\left\langle x, y \mid x^{-1} y x=y^{2 k}\right\rangle$. Производящие функции роста этих групп выгислены в [5] и при $k \geqslant 2$ имеют вид

$$
\begin{aligned}
H_{k}(z)= & \sum_{n=0}^{\infty} \delta(n) z^{n}=(1+z)(1-z)^{3}\left(1+2 z+2 z^{2}+2 z^{3}+z^{4}+2 z^{k+1}-2 z^{k+2}\right. \\
& +2 z^{k+3}+2 z^{k+4}+4 z^{k+5}-6 z^{2 k+2}-12 z^{2 k+5}+2 z^{2 k+8}-8 z^{3 k+4} \\
& -4 z^{3 k+6}-4 z^{3 k+8}+8 z^{4 k+5}+4 z^{4 k+6}-8 z^{4 k+7} \\
& \left.+4 z^{4 k+8}+8 z^{5 k+7}\right) /\left(\left(1-z-z^{2}-z^{3}+2 z^{k+2}-2 z^{k+4}\right.\right. \\
& \left.\left.+2 z^{2 k+4}\right)^{2}\left(1-2 z-z^{2}+2 z^{k+1}-2 z^{k+2}+2 z^{2 k+2}\right)\right) .
\end{aligned}
$$

Показатель роста $\lambda\left(G_{k}\right)$ групшы $G_{k}$

$$
\lambda\left(G_{k}\right)=\frac{1}{\beta_{k}}=\frac{1}{\zeta_{k}} .
$$

Здесь $\beta_{k}$ - радиус сходимости ряда Маклорена функции (6), а $\zeta_{k}-$ минимальный положительньй корень знаменателя (6). При $k \rightarrow \infty \zeta_{k} \rightarrow \zeta$, где $\zeta$ - минимальньй положительный корень функции $\psi(z)=\left(1-z-z^{2}-z^{3}\right)\left(1-2 z-z^{2}\right)$.

Нетрудно проверить, что $\zeta=-1+\sqrt{2}$ и, следовательно,

$$
\lim _{k \rightarrow \infty} \lambda\left(G_{k}\right)=\sqrt{2}+1<3 .
$$

Автор искренне благодарен Р.И. Григорчуку за постановку задачи, большую поддержку и помощь, а также И. Г. Лысенку за полезные советы и обсуждения.

\section{СПИСОК ЦИТИРОВАННОЙ ЛИТЕРАТУРЫ}

[1] Grigorchuk R. I., de la Harpe P. On problems related to growth, entropy and spectrum in group theory // J. Dynam. Control Systems. 1997. V. 3. № 1. P. 51-89.

[2] Гис Э., деля Арп П. Гиперболические группы по Михаилу Громову. М.: Мир, 1992.

[3] Линдон Р., Шупп П. Комбинаторная теория групп. М.: Мир, 1980.

[4] Григорчук Р. И. Функции роста, переписывающие системы и эйлерова характеристика // Матем. заметки. 1995. Т. 58. № 5. С. 653-668.

[5] Collins D. J., Edjvet M., Gill C. P. Growth series for the group $\left\langle x, y \mid x^{-1} y x=y^{l}\right\rangle / /$ Arch. Math. (Basel). 1997. V. 62. P. 1-11. 